\title{
Risk factors affecting COVID-19 case fatality rate: A quantitative analysis of top 50 affected countries
}

\author{
Hui Poh Goh ${ }^{1 *}$, Wafiah Ilyani Mahari ${ }^{1}$, Norhadyrah Izazie Ahad ${ }^{1}$, Li Ling Chaw ${ }^{1 *}$, Nurolaini Kifli ${ }^{1}$, Bey \\ Hing Goh ${ }^{2,3}$, Siang Fei Yeoh ${ }^{4}$, Khang Wen Goh; Long Chiau Ming ${ }^{1}$ \\ 'PAP Rashidah Sa'adatul Bolkiah Institute of Health Sciences, Universiti Brunei Darussalam, Gadong, Brunei Darussalam \\ ${ }^{2}$ College of Pharmaceutical Sciences, Zhejiang University, Hangzhou, China \\ ${ }^{3}$ Biofunctional Molecule Exploratory (BMEX) Research Group, School of Pharmacy, Monash University Malaysia, Selangor, \\ Malaysia \\ ${ }^{4}$ Department of Pharmacy, National University Health System, Singapore \\ ${ }^{5}$ Faculty of Science and Technology, Quest International University, Ipoh, Perak, Malaysia
}

\begin{abstract}
:
Background: Latest clinical data on treatment on coronavirus disease 2019 (COVID-19) indicated that older patients and those with underlying history of smoking, hypertension or diabetes mellitus might have poorer prognosis of recovery from COVID-19. We aimed to examine the relationship of various prevailing population-based risk factors in comparison with mortality rate and case fatality rate (CFR) of COVID-19.
\end{abstract}

Methods: Demography and epidemiology data were used, which have been identified as verified or postulated risk factors for mortality of adult inpatients with COVID-19. The number of confirmed cases and the number of deaths until April 16, 2020 for all affected countries were extracted from Johns Hopkins University COVID-19 websites. Datasets for indicators that are prevailing or postulated factors of COVID-19 mortality were extracted from the World Bank database. Out of 185 affected countries, the top 50 countries were selected for analysis in this study. The following seven variables were included in the analysis, based on data availability and completeness: 1) proportion of people aged 65 above, 2) proportion of male in the population, 3) smoking prevalence, and 4) number of hospital beds. Linear regression analysis was carried out to determine the relationship between CFR and the aforementioned risk factors.

Results: United States shows approximately $0.20 \%$ of confirmed cases and it has about $4.85 \%$ of CFR. Luxembourg shows the highest percentage of confirmed cases of $0.55 \%$ but a low $2.05 \%$ of CFR, showing that a high percentage of confirmed cases does not necessarily lead to high CFR. There is a significant association between CFR, people aged 65 and above $(\beta=4.70 ; p=0.035)$.

Conclusion: Countries with high proportion of older people above 65 years old have a significant risk of having high CFR from COVID-19. Nevertheless, gender differences and smoking prevalence failed to prove a significant relationship with COVID-19 mortality rate and CFR.

Keywords: COVID-19, risk, epidemiology, demography, fatality, age, diabetes

*Correspondence: Hui Poh Goh / Li Ling Chaw, Pengiran Anak Puteri Rashidah Sa'adatul Bolkiah Institute of Health Sciences Universiti Brunei Darussalam, Tungku Link Road, BE1410

Received: 19th October 2020

Accepted: 19th November 2020

Published Online: 28th November 2020 Gadong, Brunei Darussalam. Telephone: +6737213663 Email: pohhui.goh@ubd.edu.bn/ liling.chaw@ubd.edu.bn

Citation: Goh HP, Mahari WI, Ahad NI et al. Risk factors affecting COVID-19 case fatality rate: A quantitative analysis of top 50 affected countries. Prog Microbes Mol Biol 2020; 3(1): a0000171. https://doi.org/10.36877/ pmmb.a0000171.

\section{Introduction}

On February 11, 2020, the World Health Organisation (WHO) renamed the highly contagious respiratory disease, caused by severe acute respiratory syndrome coronavirus 2 (SARS-CoV-2), as coronavirus disease-19 (COVID-19) [1]. The unprecedented increase in COVID-19 cases has led WHO to call it as a pandemic on March 11, $2020^{[2,3]}$.
As this pandemic continues to evolve, researchers are learning more about SARS-CoV-2 every day, including the fact that it can be transmitted from symptomatic, presymptomatic and asymptomatic people infected with COVID-19 ${ }^{[4]}$. Studies have shown that COVID-19 is primarily transmitted from symptomatic people to people who are in close contact through respiratory droplets, by 
direct contact with infected persons or by contact with contaminated objects and surfaces ${ }^{[5]}$. Fever, tiredness and dry cough are the most common symptoms in symptomatic COVID-19 patients ${ }^{[6,7]}$.

Current evidence indicated that certain group of people are at a higher risk of suffering from severe illness from COVID-19 [8,9]. Such risk factors include gender, Bacilllus Calmette-Guerin (BCG) vaccination, smoking and malaria prevalence ${ }^{[10,11]}$. Older people have a higher risk due to the decreasing function of the agedependent lymphocytes, which resulting in their increased susceptibility to COVID-19 disease [7]. In addition, a study shows that there is a higher percentage of death among patients aged over 65 years $(62 \%)$ than patients aged below 65 years old (37\%) ${ }^{[12]}$. Furthermore, male gender is commonly observed in COVID-19 patients $(73 \%)$ according to a retrospective study done on 113 deceased patients ${ }^{[13]}$.

Another risk factor for COVID-19 mortality is existing comorbidities. A study by Guan et al. shows that COVID-19 are more commonly seen in patients with hypertension, diabetes, cardiovascular disease and a history of smoking ${ }^{[14]}$. Not only were these patients susceptible to the disease, they also had a higher chance of obtaining poor health outcomes after Immediate Care Unit (ICU) admission and may lead to death ${ }^{[12]}$. Moreover, a study on the correlation between COVID-19 mortality and BCG vaccination suggested that early BCG vaccination could help to decrease the mortality rate ${ }^{[10]}$. Other than that, malaria prevalence is also another risk factor of COVID-19 mortality. A higher number of COVID-19 cases were reported in countries with low malaria prevalence than countries that had higher malaria prevalence ${ }^{[15,16]}$. Apart from addressing risk factors, there are also parameters that may affect the COVID-19 mortality rate such as shortage of staff, lack of medical supply or equipment, insufficient hospital beds and the country's health expenditure.

As of late April 2020, SARS-CoV-2 virus has resulted in more than 3.1 million infections and over 217,000 deaths globally [1]. As COVID-19 has become a global pandemic issue, implementation of suitable interventions will be needed for the public, healthcare professionals and patients and also to ensure all sectors to work together cohesively and efficiently ${ }^{[17]}$. Even though COVID-19 originate from the same family as other known coronaviruses, SARS-CoV-2 has very different severity and contagion characteristics and much still needs to be learned about it. Thus, it is imperative to evaluate the relationship of postulated or verified risk factors with COVID-19 mortality, as presented in a recent analysis based on United Kingdom ${ }^{[18]}$ and Spain ${ }^{[19]}$. It is absolute crucial to evaluate the risk factors of mortality among patients infected with COVID-19 at population level. By validating the relationship, patients with COVID-19 can be treated more aggressively than those without the risk factor ${ }^{[20]}$. The findings of the current study provide a picture of COVID-19 case fatality rate (CFR) in top 50 affected countries.
We aimed to determine the association between specific risk factors and COVID-19 CFR. These findings consolidate the evidence of crucial risk factors that front liners need to prioritise to decrease the COVID-19 mortality globally.

This is an ecological study that examines indicators or variables that could be associated with COVID-19 mortality. The unit of observation in an ecological study is the population of the particular area or specific country in which the disease rates were measured. One of the advantages of an ecological study is it provides a snapshot of a transforming event, in additional to the fact that the disease rate and indicator statistics could be mined from existing databases thus saving time ${ }^{[21]}$. The compared populations and disease of interest are normally defined based on temporal and spatial variation. It is apparent that the reported cases of COVID-19 tend to fluctuate and the sudden spike could be linked to so local transmission cluster. In term of geographical comparisons, epidemiologists are also interested to determine the geographical associations between disease incidence or mortality and the prevalence of risk factors, as exemplified by recent COVID-19 study by Whittle and Diaz-Artiles ${ }^{[22]}$.

\section{Methods}

\section{Data extraction}

Demography and epidemiology data which have been identified as verified or postulated risk factors for mortality among adult inpatients with COVID-19 were used. The data were collected from World Bank (https:// data.worldbank.org/) and Johns Hopkins University COVID-19 (https://coronavirus.jhu.edu/map.html) websites. The number of confirmed cases and the number of deaths for all affected countries were extracted from the latter ${ }^{[23]}$, while datasets for indicators that are prevailing or postulated as the risk factors of COVID-19 mortality were extracted from the World Bank database ${ }^{[24]}$. Data extracted for this study was up until April 16, 2020. All data acquired were exported in excel format and arranged according to country rankings with the top having the highest number of confirmed cases as of April 16, 2020 and the bottom having the least. To facilitate comparison, out of about 185 affected countries, only top 50 countries were selected to be analyzed in this study.

The following seven variables were included in the analysis, based on data availability and completeness: 1) proportion of people aged 65 above, 2) proportion of male in the population, 3) smoking prevalence, and 4) number of hospital beds.

\section{Data analysis}

For each country, the percentage of confirmed COVID-19 case per country was calculated by dividing the number of confirmed COVID-19 cases by the total population for each country. Also, CFR was calculated by dividing the number of deaths related to COVID-19 by the confirmed COVID-19 cases.

Linear regression analysis was conducted to determine the risk factors of CFR for COVID-19. This method was 
chosen so that the degree of association can be quantified and that this estimate can be adjusted with other potential risk factors. For this analysis, two variables (CFR and number of hospital beds) were standardized due to differences in scale and very large range. Standardization was done by subtracting each value by the mean and then dividing it with the standard deviation. However, data for four variables (diabetes prevalence, current health expenditure, and number of nurses and midwives) were not normally distributed. Transforming these variables to normality were performed but model over-fitting occurred, therefore further analysis was not pursued. All analyses were conducted using Microsoft Excel and R (ver. 3.6.0). A $p$-value $<0.05$ was considered as statistically significant.

\section{Results}

Information of 2,017,444 confirmed COVID-19 cases and 137,166 deaths from each of the 50 top countries (Supplementary Information) were extracted. This constitutes about $93.3 \%$ and $92.8 \%$ of the global confirmed cases and and deaths on the data collection date (April 16, 2020).

\section{Case Fatality Rate (CFR) and mortality rate of COVID-19 in the top 50 countries}

From data extracted up until April 16, 2020, the United States (US) reported to have the highest number of total confirmed cases and the highest total number of deaths of 639,733 cases and 31,002 cases respectively. Despite that, the US accounted about $0.20 \%$ (Table 1) of confirmed cases and it had about $4.85 \%$ of CFR (Table 1), indicating a moderate mortality rate of COVID-19 in comparison to other countries. Luxembourg, ranked $47^{\text {th }}$ in the list of top 50 COVID-19 countries, had the highest percentage of confirmed cases of $0.55 \%$ but a low $2.05 \%$ of CFR (Table 1 ), indicating that a high percentage of confirmed cases does not necessarily lead to a high CFR. This is due to variations in number, transmission rate and severity of the disease regardless of the rankings ${ }^{[25]}$. Hence, it is important to evaluate the possible factors that can affect the increase of COVID-19 mortality rate globally.

Table 1: Percentage of confirmed CFR and COVID-19 cases (In sequence of the highest to lowest CFR\%)

\begin{tabular}{|c|c|c|c|}
\hline No. & Countries & $\begin{array}{c}\text { Confirmed COVID-19 cases } \\
(\%)\end{array}$ & Case Fatality Rate (\%) \\
\hline 1 & Belgium & 0.3 & 13.95 \\
\hline 2 & United Kingdom & 0.15 & 13.82 \\
\hline 3 & Italy & 0.27 & 13.11 \\
\hline 4 & France & 0.2 & 12.77 \\
\hline 5 & Netherlands & 0.17 & 11.32 \\
\hline 6 & Sweden & 0.12 & 10.63 \\
\hline 7 & Spain & 0.39 & 10.46 \\
\hline 8 & Indonesia & 0.002 & 8.99 \\
\hline 9 & Mexico & 0.005 & 7.68 \\
\hline 10 & Philippines & 0.01 & 6.4 \\
\hline 11 & Iran & 0.1 & 6.24 \\
\hline 12 & Brazil & 0.01 & 6.07 \\
\hline 13 & Dominican Republic & 0.03 & 5.23 \\
\hline 14 & Romania & 0.04 & 5.09 \\
\hline 15 & Ecuador & 0.05 & 4.94 \\
\hline 16 & United States & 0.2 & 4.85 \\
\hline 17 & Switzerland & 0.31 & 4.8 \\
\hline 18 & Denmark & 0.12 & 4.54 \\
\hline 19 & Colombia & 0.01 & 4.22 \\
\hline 21 & Poland & 0.02 & 3.76 \\
\hline 22 & Canada & 0.08 & 3.56 \\
\hline 23 & Ireland & 0.26 & 3.54 \\
\hline 24 & India & 0.001 & 3.4 \\
\hline 25 & Portugal & 0.18 & 3.33 \\
\hline 26 & Germany & 0.1 & 2.86 \\
\hline 27 & Ukraine & 0.01 & 2.79 \\
\hline
\end{tabular}


Table 1: Percentage of confirmed CFR and COVID-19 cases (In sequence of the highest to lowest CFR\%) (Continued)

\begin{tabular}{|c|c|c|c|}
\hline 28 & Panama & 0.09 & 2.75 \\
\hline 29 & Austria & 0.16 & 2.73 \\
\hline 30 & Czech Republic & 0.06 & 2.63 \\
\hline 31 & Finland & 0.06 & 2.23 \\
\hline 32 & Norway & 0.13 & 2.21 \\
\hline 33 & Peru & 0.04 & 2.21 \\
\hline 34 & Turkey & 0.08 & 2.19 \\
\hline 35 & Korea, Rep & 0.02 & 2.16 \\
\hline 36 & Japan & 0.01 & 2.06 \\
\hline 37 & Luxembourg & 0.56 & 2.05 \\
\hline 38 & Serbia & 0.07 & 2.03 \\
\hline 39 & Pakistan & 0.003 & 1.85 \\
\hline 40 & Thailand & 0.004 & 1.72 \\
\hline 41 & Malaysia & 0.02 & 1.62 \\
\hline 42 & Saudi Arabia & 0.02 & 1.3 \\
\hline 43 & Chile & 0.04 & 1.14 \\
\hline 44 & Israel & 0.14 & 1.11 \\
\hline 45 & Australia & 0.03 & 0.97 \\
\hline 46 & Belarus & 0.04 & 0.95 \\
\hline 47 & Russian Federation & 0.02 & 0.83 \\
\hline 48 & United Arab Emirates & 0.06 & 0.62 \\
\hline 49 & Singapore & 0.07 & 0.27 \\
\hline 50 & Qatar & 0.15 & 0.17 \\
\hline
\end{tabular}

\section{Relationship between the different risk factors and COVID-19 CFR}

The proportion of people aged 65 and above had a significant association with CFR (4.70 [95\% CI: 0.34,
9.06], $p=0.04$, Table 2). For every 1 -unit increase in the proportion of people aged 65 years, the CFR increased by 4.7 units. This relationship is illustrated in Figure 1, where CFR sharply increases when the proportion of people aged 65 years is 0.18 and above.

Table 2: Linear regression analysis for different risk factors (independent variable) and COVID-19 CFR (dependent variable)

\begin{tabular}{ccc}
\hline Independent variable & $\beta$ estimate $(95 \% \mathrm{Cl})$ & Standard Errors \\
\hline Age (above 65 years) & $4.70(0.34,9.06)$ & 2.17 \\
\hline Gender - Male & $-0.33(-0.92,0.26)$ & 0.29 \\
\hline Smoking Prevalence (total \% of people aged 15 & $0.009(-0.03,0.04)$ & 0.02 \\
and above) & & 0.60 \\
\hline Hospital Beds (per 1,000 people) & & 0.14 \\
\hline
\end{tabular}

\section{Discussion}

There are still a lot of unknown regarding COVID-19 disease. However, good clinical findings are made available now to understand the risk factors that could affect its treatment outcomes.
Studies have shown that age is a clear risk factor for severe COVID-19 disease. This has been confirmed by our study where the proportion of people aged 65 and above has shown a significant association with CFR. This indicates that countries with a higher proportion of people aged 65 and above may result in higher COVID-19 mortality 


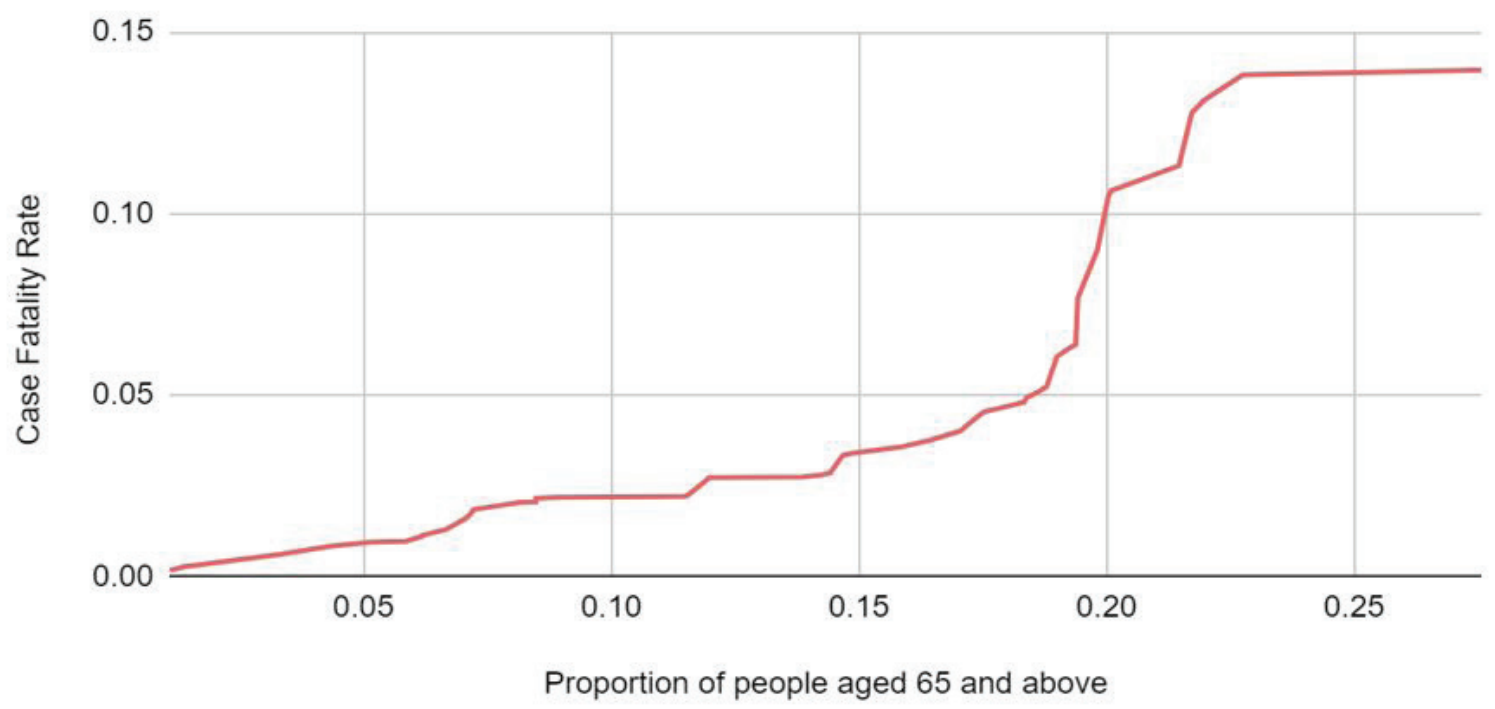

Figure 1: Association between case fatality rate and proportion of people aged 65 and above.

rate (Figure 1). Bhatraju et al. (2020) has shown that in Seattle, the US reported more than $60 \%$ of COVID-19 deaths in patients aged 65 years and above than those who are younger than 65 years old ${ }^{[12]}$. Verity et al. (2020) has shown that the a stark difference in the CFR between those aged below and above 60 years $(1.4 \%$ versus $4.5 \%$, respectively) ${ }^{[9]}$. This suggests that the older the country population, the higher the CFR. For those 80 years old and above, COVID-19 appears to have a $13.4 \%$ fatality rate ${ }^{[9]}$. Notably, the average age of deceased and recovered patients were found to be 68 and 51 years, respectively ${ }^{[13]}$. These studies show that COVID-19 disproportionately impacts certain groups, and that older people ${ }^{[26]}$ and pregnant women [27] are among the vulnerable groups. This could be due to the weakening effects of ageing on the immune system. As age increases, there is an increase of deficiency in T-cell and B-cell function and overproduction of type 2 cytokines ${ }^{[7]}$. This may promote viral replication and extend the duration of pro-inflammatory responses, leading to poor prognosis ${ }^{[7]}$. Furthermore, older people tend to have more underlying conditions that may also be risk factors for severe COVID-19 ${ }^{[9,14]}$.

Studies have shown that many of the severe COVID-19 patients also have underlying medical conditions, such as diabetes and cardiovascular diseases ${ }^{[13,28]}$. Patients with existing comorbidities, including hypertension, diabetes, cardiovascular disease and history of smoking, seems to be associated with COVID-19 more severely [14]. With reference to a retrospective study of 113 deceased patients from COVID-19, $48 \%$ of the patients had chronic hypertension and $14 \%$ of them had cardiovascular diseases ${ }^{[13]}$. In addition to that, COVID-19 patients who have hypertension were closely associated with poor health outcomes after hospital admission. This may be due to factors such as vascular aging, reduced renal function and medication interactions ${ }^{[29]}$. Although smoking prevalence has shown no significant association with COVID-19 $(p=0.60)$, it cannot be assumed that there is no association between other comorbidities and COVID-19 CFR since not all factors were considered in this study, such as hypertension and cardiovascular diseases ${ }^{[6]}$.
COVID-19 is a rapid spreading communicable disease and general public are responsible in controlling this COVID-19 pandemic. Various levels of wellpreparedness plans are needed to tackle this pandemic situation ${ }^{[30]}$. These include availability of medicines or medical supplies to treat COVID-19 patients, availability of suitable places to quarantine or self-isolate these patients, number of healthcare professionals and a strategized interventions, such as social distancing, quarantine, isolation actions and proper management, for the patients and public to flatten the curve and to reduce healthcare burden. It is necessary for some countries which may need more supplies than others to cater all sick patients, and thus increasing the health expenditure of the country ${ }^{[2]}$. Some of the medical supplies include personal protective equipment, mechanical ventilators, COVID-19 testing kits and extracorporeal membrane oxygenation. Due to surge of demand on healthcare system, countries with low income and poor healthcare infrastructure suffer the most ${ }^{[14]}$. However, in our study, current health expenditure of the top 50 countries was not tested due to aforementioned reason.

Sufficient hospital capacity in term of hospital bed is necessary to accommodate unforeseen pandemic situations [31]. Despite the insignificant association to COVID-19 CFR $(p=0.50)$, accessibility to adequate hospital beds for COVID-19 patients can potentially affect the CFR as the number of beds required depends on the number of confirmed cases in each country. Apart from hospital beds, other medical supplies such as medical grade face mask and mechanical ventilators must be sufficient as they are the key equipment for frontline healthcare workers ${ }^{[32-34]}$.

Evidence of high numbers of infections and deaths among healthcare workers due to lack of face mask and medical gowns were reported in Italy ${ }^{[32]}$. In the US, recent estimates have suggested that the estimated ventilators needed is ranging from several hundred thousand to a million, far more than what are currently available ${ }^{[32]}$. It is difficult to estimate the exact number of ventilators needed as it depends on the number, transmission rate and severity of the disease in each country. 
There are a number of limitations in this study. The nature of the study design (ecological study) and type of analysis used (linear regression analysis) allowed us to use countrylevel aggregated data to determine the relationship between CFR and specific risk factors. Since aggregated data were used, this means that the results are only applicable at a country-level, instead of individual-level. Some factors had to be excluded due to incomplete data such as malaria prevalence and BCG vaccination. Even if the data were available, the data may come from different years. The years from which the data were retrieved were not consistent for all indicators. The data collected were also limited by unavailability of certain data to sufficiently make an overall conclusion for several factors, including comorbidities. There were four other proposed comorbidities to be analyzed but only two indicators' datasets were available in World Bank Data, which are diabetes and smoking prevalence. Of note, our study did not able to test the association between diabetes prevalence and CFR. Therefore, more research should be conducted to further understand the relationship between comorbidities and CFR. This would help to identify and to better understand other possible factors that may also affect CFR. However, even with these limitations, it is important to note that the aim of our report is to determine the association between specific risk factors and COVID-19 case fatality rate globally. Also, our analysis is limited by data derived from confirmed COVID-19 cases as of April 16, 2020, yet the number of global cases continues to increase. It is also important to note that transmissibility rate and various periods of the pandemic were not taken into account, as it may differ at different countries or even area within the same country ${ }^{[6,35,36]}$.

\section{Conclusion}

As COVID-19 is such a new disease, much still needs to be learned about it. Age is a clear risk factor for severe COVID-19 and death. COVID-19 is an illness that disproportionately impacts older people. However, other risk factor such as smoking should not be neglected. Prediction alone is not efficient, but well-planned and suitable interventions should also be carried out. In addition to that, potential risk factors need a lot more research in order to understand the risks for the worst forms of COVID-19 and what we ought to learn to best protect the people with higher risk. Participation and involvement of every individual, including patients, public and healthcare professionals, is necessary and everyone should work together towards combating COVID-19 disease.

\section{Conflict of interest}

None declared.

\section{Funding}

None.

\section{Author contributions}

LCM conceived the project. HPG, WIM, NIA, LLC, LCM analyzed results and interpreted the data and wrote the manuscript draft. NK, BHG, KWG, SFY revised the manuscript. All authors read and approved the final manuscript.

\section{References}

1. World Health Organization Novel Coronavirus (2019-nCoV) Situation Report - 22. Accessed from https://www. who. int/docs/default-source/coronaviruse/situation-reports/20200211-sitrep-22-ncov. pdf [Internet].

2. Bedford J, Enria D, Giesecke J, et al. COVID-19: towards controlling of a pandemic. Lancet 2020; 395: 1015-1018.

3. Johnson D, Ren SEC, Johnson HD, et al. COVID-19: Are Malaysians embracing or suffering the new normality? Prog Microbes Mol Biol 2020; 3: a0000102. doi: 10.36877/pmmb.a0000102

4. Tan CS, Ming LC, Yeoh SF. COVID-19: Critical role of angiotensin 1-7 in ACE2 modulation. Ann Acad Med Singapore 2020; 49: 398-400.

5. Koh D, Goh HP. Occupational health responses to COVID-19: What lessons can we learn from SARS? J Occup Health 2020; 62: e12128.

6. Yang J, Zheng Y, Gou X, et al. Prevalence of comorbidities and its effects in coronavirus disease 2019 patients: A systematic review and meta-analysis. Int J Infect Dis 2020; 94: 91-95. doi:10.1016/j. ijid. 2020.03.017.

7. Zhou F, Yu T, Du R, et al. Clinical course and risk factors for mortality of adult inpatients with COVID-19 in Wuhan, China: a retrospective cohort study. Lancet 2020; 395: 1054-1062. doi:10.1016/ S0140-6736(20)30566-3.

8. Siddiqi HK and Mehra MR. COVID-19 Illness in Native and Immunosuppressed States: A Clinical-Therapeutic Staging Proposal. J Hear Lung Transplant 2020; 39: 405. doi:10.1016/j.healun.2020.03.012.

9. Verity R, Okell LC, Dorigatti I, et al. Estimates of the severity of coronavirus disease 2019: a model-based analysis. Lancet Infect Dis 2020; 20: 669-677. doi:10.1016/S1473-3099(20)30243-7.

10. Miller A, Reandelar MJ, Fasciglione K, et al. Correlation between universal BCG vaccination policy and reduced mortality for COVID-19: an epidemiological study. MedRxiv 2020. doi:10.1101/2020.03.24.20042937.

11. Moran KR and Del Valle SY, A meta-analysis of the association between gender and protective behaviors in response to respiratory epidemics and pandemics. PLoS One 2016; 11: e0164541. doi:10.1371/ journal. pone. 0164541

12. Bhatraju PK, Ghassemieh BJ, Nichols M, et al. Covid-19 in Critically Ill Patients in the Seattle Region - Case Series. N Engl J Med 2020; 382: 2012-2022. doi:10.1056/nejmoa2004500.

13. Chen T, Wu D, Chen $\mathrm{H}$, et al. Clinical characteristics of 113 deceased patients with coronavirus disease 2019: Retrospective study. BMJ 2020; 368. doi:10.1136/bmj.m1091.

14. Guan W, Ni Z, Hu Y, et al. Clinical characteristics of 2019 novel coronavirus infection in China. N Engl J Med 2020; 382: 1708-1720. doi:10 . $1101 / 2020.02 .06 .20020974$

15. Some COVID-19 vs. Malaria Numbers: Countries with Malaria have Virtually no Coronavirus Cases Reported « Roy Spencer, PhD.

16. Napoli PE and Nioi M. Global Spread of Coronavirus Disease 2019 and Malaria: An Epidemiological Paradox in the Early Stage of A Pandemic. J Clin Med 2020; 9. 1138 doi: $10.3390 / \mathrm{jcm} 9041138$

17. Ming LC, Untong N, Aliudin NA, et al. Mobile Health Apps on COVID-19 Launched in the Early Days of the Pandemic: Content Analysis and Review. JMIR mHealth uHealth 2020; 8: e19796. doi:10.2196/19796.

18. Tan LTH, Letchumanan V, Ser HL, et al. PMMB COVID-19 Bulletin: United Kingdom (22nd April 2020). Prog Microbes Mol Biol 2020; 3. doi: 10.36877/pmmb.a0000078

19. Ser HL, Letchumanan V, Law JWF, et al. PMMB COVID-19 Bulletin: Spain (18th April 2020). Prog Microbes Mol Biol 2020; 3. doi:10.36877/ pmmb.a0000074

20. Cunningham AC, Goh HP, Koh D. Treatment of COVID-19: Old tricks for new challenges. Crit Care 2020; 24: Article number 91. doi: 10.1186/ s13054-020-2818-6

21. Coggon D, Rose G, Barker D. Chapter 6. Ecological studies| The BMJ. In Epidemiology for the uninitiated; 2003.

22. Whittle RS and Diaz-Artiles A. An ecological study of socioeconomic predictors in detection of COVID-19 cases across neighborhoods in New York City. MedRxiv 2020; doi:10.1101/2020.04.17.20069823.

23. Novel Coronavirus (COVID-19) Cases Data - Humanitarian Data Exchange.

24. World Bank Open Data $\mid$ Data.

25. Wu JT, Leung K, Bushman M, et al. Estimating clinical severity of COVID-19 from the transmission dynamics in Wuhan, China. Nat Med 2020; 26: 506-510. doi:10.1038/s41591-020-0822-7.

26. Bonafè M, Prattichizzo F, Giuliani A, et al. Inflamm-aging: Why older men are the most susceptible to SARS-CoV-2 complicated outcomes. Cytokine Growth Factor Rev 2020; 53: 33-37. doi:10.1016/j.cytogfr. 2020.04.005.

27. Phoswa WN and Khaliq OP. Is pregnancy a risk factor of COVID-19? Eur J Obstet Gynecol Reprod Biol 2020; 252: 605-609.

28. Huang C, Wang Y, Li X, et al. Clinical features of patients infected with 2019 novel coronavirus in Wuhan, China. Lancet 2020; 395: 497-506, doi:10.1016/S0140-6736(20)30183-5.

29. Coronavirus Disease 2019 (COVID-19): Epidemiology, Clinical Spectrum and Implications for the Cardiovascular Clinician - American College of Cardiology.

30. Letchumanan V, Ab Mutalib NS, Goh BH, et al. Novel coronavirus 2019-nCoV: Could this virus become a possible global pandemic. Prog Microbes Mol Biol 2020; 3: 1-6, doi:10.36877/pmmb. a0000068. 
31. Hick JL and Biddinger PD. Novel Coronavirus and Old Lessons - Preparing the Health System for the Pandemic. N Engl J Med 2020; 382: e55. doi:10.1056/NEJMp2005118.

32. Ranney ML, Griffeth V, Jha AK. Critical Supply Shortages - The Need for Ventilators and Personal Protective Equipment during the Covid-19 Pandemic. N Engl J Med 2020; 382: e41. doi: 10.1056/ NEJMp2006141.

33. Adams JG and Walls RM. Supporting the Health Care Workforce during the COVID-19 Global Epidemic. JAMA - J Am Med Assoc 2020; 323: $1439-1440$.
34.

Liu Q, Yang J, Liu Q, et al. The experiences of health-care providers during the COVID-19 crisis in China: a qualitative study. Lancet Glob Heal 2020; 8: e790-e798. doi:10.1016/S2214-109X(20)30204-7.

35. Deng SQ and Peng HJ. Characteristics of and Public Health Responses to the Coronavirus Disease 2019 Outbreak in China. J Clin Med 2020; 9: 575. doi:10.3390/jcm9020575.

36. Shim E, Tariq A, Choi W, et al Transmission potential and severity of COVID-19 in South Korea. Int J Infect Dis 2020; 93: 339-344. doi: 10.1016/j.ijid.2020.03.031. 